\section{Visualização dos padrões de variação da taxa de mortalidade infantil no Rio Grande do Sul, Brasil: comparação entre as abordagens Bayesiana Empírica e Totalmente Bayesiana}

\author{
Patterns of variation in the infant mortality rate \\ in Rio Grande do Sul State, Brazil: comparison of \\ empirical Bayesian and fully Bayesian approaches
}

\author{
1 Programa de Pós-graduação \\ em Epidemiologia \\ Universidade Federal do Rio \\ Grande do Sul, Porto Alegre, \\ Brasil. \\ 2 Instituto Federal de \\ Educação, Ciência e \\ Tecnologia do Rio Grande do \\ Sul, Porto Alegre, Brasil. \\ 3 Instituto de Matemática, \\ Universidade Federal do Rio \\ Grande do Sul, Porto Alegre, \\ Brasil. \\ 4 Departamento de Educação \\ e Informação em Saúde, \\ Universidade Federal de \\ Ciências da Saúde de Porto \\ Alegre, Porto Alegre, Brasil. \\ 5 Faculdade de Matemática, \\ Pontifícia Universidade \\ Católica do Rio Grande do \\ Sul, Porto Alegre, Brasil. \\ 6 Faculdade de Medicina, \\ Universidade Federal do Rio \\ Grande do Sul, Porto Alegre, \\ Brasil. \\ Correspondência \\ S. L. C. Silva \\ Programa de Pós-graduação \\ em Epidemiologia, \\ Universidade Federal do Rio \\ Grande do Sul. \\ Rua Ramiro Barcelos 2777 \\ Porto Alegre, RS 90035-007, \\ Brasil. \\ sassalelenash@yahoo.com.br
}

\begin{abstract}
Infant mortality is considered a sensitive health indicator, and knowledge of its geographical profile is essential for formulating appropriate public health policies. Disease mapping aims to describe the geographical distribution of disease incidence and mortality rates. Due to the heavy instability of crude rates in small areas, methods involving Bayesian smoothing of rates are used, drawing on information for the whole area or neighborhood to estimate the event rate. The current study compares empirical Bayesian (EB) and fully Bayesian (FB) methods for infant mortality rates (accumulated data from 2001 to 2004) in Rio Grande do Sul State, Brazil. This study highlights the advantages of Bayesian estimators for viewing and interpreting maps. For the problem at hand, EB and FB methods showed quite similar results and had the great advantage of easy use by health professionals, since they evenly highlight the main spatial patterns in the mortality rate in the State during the target period.
\end{abstract}

Infant Mortality; Mortality Rate; Risk Map
Sabrina Letícia Couto da Silva 1,2 Jandyra Maria Guimarães Fachel 3 Sergio Kakuta Kato ${ }^{4,5}$ Sergio Luiz Bassanesi 6

\section{Introdução}

A modelagem estatística espacial tem sido bastante utilizada em diversos ramos da ciência nos quais os dados são coletados em diferentes locais, isto é, cada observação está associada a um ponto ou a uma região no espaço. Os dados podem ser pontuais, quando a exata localização de sua ocorrência é conhecida, ou contagens por área, quando os dados são agregados em áreas geograficamente definidas.

Quando os dados são contagens por áreas, o tamanho da população em risco pode variar consideravelmente entre as áreas sob estudo. A dispersão espacial do risco de ocorrência de um evento (por exemplo, doença, morte etc.) é usualmente mostrada via mapas de incidência de taxas. O objetivo deste mapeamento é inferir a respeito da distribuição geográfica das taxas e então identificar as áreas de maior ou menor incidência, buscando um padrão espacial. Quando a autocorrelação está presente nos dados, regiões próximas tendem a ter um mesmo padrão de ocorrência do evento de interesse e regiões distantes terão padrões distintos. Um exemplo muito usado é o mapeamento das taxas de mortalidade. A taxa de mortalidade infantil (TMI) reflete os níveis de saúde e de desenvolvimento socioeconômico de uma determinada área, sendo considerado um dos mais importantes indicadores epidemiológicos utilizados ${ }^{1}$ e também tem sido utilizada como um dos principais 
indicadores da qualidade de vida de uma população 2,3. A taxa é dada pela relação entre o número de óbitos de crianças menores de um ano e o número de nascidos vivos, em determinado local e período, calculado na base de mil nascidos vivos. A TMI é um importante indicador, porque reflete a qualidade com os cuidados pré e pós-natal das crianças, além de indicar uma preocupação na redução das disparidades existentes na pirâmide social.

Conhecer o perfil da mortalidade infantil é fundamental para a formulação de estratégias que permitam seu controle, como uma assistência adequada à mulher durante a gravidez e o parto e, principalmente, um acompanhamento cuidadoso das mães em gestação considerada de risco. Segundo dados da Secretaria Estadual de Saúde do Rio Grande do Sul - SES-RS (http:// www.saude.rs.gov.br, acessado em 10/Jun/2009), a TMI no estado para o ano de 2008 foi calculada em 12,8 óbitos por mil nascidos vivos. Para o Brasil, segundo os indicadores de mortalidade do Instituto Brasileiro de Geografia e Estatística - IBGE (http://www.datasus.gov.br, acessado em 10/Jun/2009), a TMI para o ano de 2006 foi estimada em 20,7 óbitos por mil nascidos vivos. Analisando os dados historicamente, percebese uma redução significativa na TMI estadual e também uma redução no indicador brasileiro 4 . Ainda segundo os mesmos autores, o declínio da mortalidade infantil tem sido atribuído a vários fatores, tais como: intervenções ambientais, avanços da medicina, expansão do acesso a cuidados de saúde, diminuição da taxa de fecundidade, aumento do nível educacional da população e melhoria nutricional e das condições de vida em geral.

Dentre as alternativas conhecidas, cálculos baseados na taxa bruta são os mais simples para o estudo da ocorrência de um evento, sendo definida como uma razão entre o número de eventos ocorridos e o número de pessoas expostas à ocorrência desse evento em uma determinada localidade. Entretanto, existe um grande problema relacionado ao uso de taxas brutas que é a sua alta instabilidade para medir o risco de um evento quando o evento é raro ou quando a população da região de ocorrência é pequena. Dessa forma, a alta variabilidade das taxas estimadas pode não estar diretamente relacionada com o fenômeno investigado, mas sim com uma variabilidade associada aos dados. Instabilidades como a ocorrência de um ou dois casos do evento a mais ou a menos numa localidade tem um impacto grande no cálculo das taxas brutas se a população de interesse for pequena, efeito que não se verifica em localidades de população de tamanho grande.
Realizando a análise da distribuição espacial de eventos por mapas construídos com base nas estimativas brutas, frequentemente, a interpretação se torna mais difícil e pode gerar conclusões errôneas 5 . Para corrigir a instabilidade das taxas brutas, existem diversas técnicas de suavização como os métodos baseados na teoria bayesiana, que estão sendo utilizados com maior frequência em estudos epidemiológicos espaciais. Esses estudos objetivam mapear algum evento de interesse e estudar sua distribuição espacial, bem como analisar fatores que possam estar associados a sua ocorrência no espaço-tempo.

Dentre as abordagens bayesianas existentes para suavização de taxas, destacam-se a Bayesiana Empírica e a Totalmente Bayesiana. O crescimento do uso dessas técnicas se deve ao grande avanço nos recursos computacionais disponíveis e também à necessidade de se obter estimativas mais suavizadas, que reflitam de forma mais adequada a característica subjacente do evento de interesse, sem a influência da alta variabilidade observada nos dados brutos 6 .

A abordagem Totalmente Bayesiana utiliza informações $a$ priori e mediante a informação das áreas vizinhas geograficamente geram distribuições a posteriori que serão usadas para as inferências sobre os parâmetros de interesse. Em geral, a distribuição a posteriori possui uma forma bastante complexa e muitas vezes não é possível obter sua derivação via cálculos analíticos. Tal problema é solucionado pelo uso da metodologia de simulação MCMC (Markov Chain Monte Carlo) 7. O método é teoricamente preferível, pois pode ser generalizado para modelos mais complexos, apesar do enorme esforço computacional necessário para sua aplicação. Para resolver o problema da dificuldade de implementação computacional que a metodologia MCMC envolve, novas ferramentas vêm sendo desenvolvidas, como o pacote R-INLA 8 , que utiliza o método Integrated Nested Laplace Approximation (INLA). Alguns estudos vêm usando essa nova abordagem para realização de inferência em modelos dinâmicos bayesianos para processos pontuais espaço-temporais ao invés da abordagem usual que utiliza a MCMC 9. Alternativamente, há o método Bayesiano Empírico, que se destaca por utilizar os dados observados para estimar os parâmetros do a priori 10 .

Em um estudo comparativo entre as duas abordagens, os métodos Bayesianos Empíricos apresentaram resultados muito semelhantes àqueles apresentados pelos métodos Totalmente Bayesianos e, ainda, possuem a grande vantagem de ser de fácil integração aos ambientes de geoprocessamento 11. Os estimadores Bayesianos Empíricos têm sido bastante úteis para 
fornecer padrões do risco da doença no contexto dos Sistemas de Informações Geográfica (SIG) para epidemiologia espacial, no qual grandes quantidades de dados estão disponíveis e os algoritmos usados devem ser rápidos. Os métodos Bayesianos Empíricos são muito mais fáceis de serem utilizados do que os métodos Totalmente Bayesianos e estão implementados em aplicativos de geoprocessamento como o TerraView, desenvolvido pelo INPE (Instituto Nacional de Pesquisas Espaciais; http://www.dpi.inpe.br/ terraview), podendo ser utilizados facilmente pelos profissionais das secretarias municipais e estaduais de saúde. Trata-se de um programa livre com uma interface bastante amigável. Ugarte et al. 5 fizeram um comparativo entre os métodos Bayesiano Empírico e Totalmente Bayesiano e mostraram que, em casos em que se deseja identificar regiões de elevado risco, o excesso de suavização pode ser um problema por não detectar regiões de risco potencial para o evento analisado. Como as estimativas obtidas pelos dois métodos foram similares, a abordagem Bayesiana Empírica seria conveniente porque utiliza a técnica de Quase-verossimilhança Penalizada (PQL) 12, que é considerada bastante simples e requer menos recursos computacionais, tanto em capacidade computacional instalada, como no tempo computacional necessário. Quando o risco subjacente é elevado e a população é não dispersa, a abordagem Bayesiana Empírica é mais adequada para suavizar os riscos estimados e detectar áreas de alto risco 13.

O trabalho de Aimsworth \& Dean 14 efetuou a comparação entre as abordagens Bayesiana Empírica - mediante o uso da técnica de PQL com a Totalmente Bayesiana - pelo emprego da metodologia MCMC. Os autores mostram que os resultados das duas técnicas foram bastante similares.

Vieira ${ }^{15}$, Kato 16 e Kato et al. 17, em uma sequência de trabalhos abordando a mortalidade infantil no Rio Grande do Sul, utilizaram o método de estimação Totalmente Bayesiano. Vieira 15 utilizou a metodologia de análise Espaço-Temporal Bayesiana para estimação do risco de mortalidade infantil em cada município gaúcho para dados de 2001 a 2004. A metodologia utiliza a informação a priori tanto dos municípios vizinhos, como de anos próximos. Kato 16 e Kato et al. 17 utilizaram alguns modelos de regressão espacial bayesianos para estimação do risco relativo com a inclusão de covariáveis socioeconômicas nos modelos para dados do mesmo período. O presente trabalho pertence a essa série de estudos sobre a metodologia Bayesiana para Mapeamento de Doenças em Epidemiologia Espacial e introduz resultados obtidos pelo do método Baye- siano Empírico, comparando-os com o método Totalmente Bayesiano.

O objetivo do artigo é comparar as estimativas para as TMI nos municípios do Rio Grande do Sul, geradas pelos métodos: Clássico (Taxa Bruta), Bayesiano Empírico Local (BEL), Bayesiano Empírico Global (BEG) e Totalmente Bayesiano (TB), utilizando os dados acumulados dos anos 2001 a 2004. A análise será feita por intermédio do cálculo de estatísticas descritivas e representação gráfica para visualizar o comportamento das estimativas geradas por cada método e por comparação dos resultados por Coordenadorias Regionais de Saúde (CRS). Finalmente, também foram gerados os mapas das taxas estimadas para identificação do padrão espacial subjacente dos dados.

\section{Metodologia}

Foi realizado um estudo epidemiológico ecológico, do tipo análise espacial em saúde. As unidades de análise são os 496 municípios do Estado do Rio Grande do Sul existentes no período de 2001 a 2004. As informações sobre o número de nascimentos e óbitos em recém-nascidos em cada município foram obtidas originalmente no Departamento de Informática do SUS (DATASUS) e são as mesmas utilizadas por Vieira 15, Kato 16 e Kato et al. 17 para fins de comparação dos métodos. A base de dados com o georreferenciamento dos municípios foi fornecida pelo Centro Estadual de Vigilância em Saúde (CEVS) da SES-RS, possibilitando a construção dos mapas das taxas estimadas. Foram calculadas as TMI brutas acumuladas para o período de 2001 a 2004. A taxa acumulada foi calculada pela utilização do total dos óbitos no período dividido pelo total de nascidos vivos no período.

Segundo Marshall 18, denotando por $\hat{\theta}$ a estimativa Bayesiana Empírica de certa área, por $t$ a taxa bruta dessa área e por $m$ a taxa média de todas as áreas, podemos escrever,

$$
\hat{\theta}=m+c(t-m)
$$

O valor de $c$ está entre 0 e 1 e representa o peso dado à diferença entre a taxa bruta da área e a taxa média de todas as áreas. O cálculo de $c$ leva em conta o tamanho da população da área. Quando essa população for relativamente grande, o valor de $c$ é próximo de 1 , tornando a estimativa $\hat{\theta}$ muito próxima da taxa bruta, que, nessa área, não sofre o problema da instabilidade. No entanto, quando a população for pequena, o valor de $c$ decresce, dando pouco peso à diferença entre $t$ e $m$ e tornando a estimativa $\hat{\theta}$ mais próxima da taxa média de todas as áreas. Ou seja, quando a 
população da área é pequena, a informação contida na taxa média tem peso maior na estimativa Bayesiana Empírica. A expressão para $\hat{\theta}$ também pode ser escrita como,

$$
\hat{\theta}=c t+(1-c) m,
$$

em que se nota que a estimativa $\hat{\theta}$ é a média ponderada entre a taxa bruta da área e a taxa média de todas as áreas, ponderada pelo valor $c$.

O estimador Bayesiano Empírico que utiliza como valor de $m$ a taxa média de todas as áreas em estudo é denominado estimador BEG. O estimador BEG calcula uma média ponderada entre a taxa bruta da localidade e a taxa global da região (razão entre o número total de casos e a população total). Existe ainda o estimador BEL que inclui o efeito espacial, calculando a estimativa localmente, utilizando somente os vizinhos geográficos da área na qual se deseja estimar a taxa: o valor obtido converge em direção à média local ao invés de uma média global. As taxas corrigidas são menos instáveis, pois levam em conta no seu cálculo não só a informação da área, mas também a informação de sua vizinhança. Mapas baseados nessas estimativas são mais interpretativos e informativos 19.

Na abordagem Totalmente Bayesiana, supõese que o número de eventos observados em cada área possui distribuição binomial, mas como a maioria dos dados epidemiologicamente mapeados é rara ou com grande variação das taxas entre diferentes áreas, o modelo binomial pode ser aproximado pela distribuição Poisson 20. Assim, se o número de eventos observados (Yi) se refere ao número de óbitos no município i,

$$
\mathrm{Y}_{i} \sim \operatorname{Poisson}\left(\mu_{\mathrm{i}}\right),
$$

onde $\mu_{\mathrm{i}}=\mathrm{E}_{\mathrm{i}} \theta_{\mathrm{i}}$, sendo $\theta_{\mathrm{i}}$, o risco relativo de óbito infantil na área i e $\mathrm{E}_{\mathrm{i}}$, a quantidade esperada de óbitos infantis na i-ésima área sob a hipótese de que o risco seja constante em todas as áreas e igual ao risco geral da região. Sendo assim, o primeiro nível hierárquico do modelo é dado por:

$$
\mathrm{Y}_{i} \mid \mathrm{E}_{i} \sim \operatorname{Poisson}(\mathrm{Ei} \theta \mathrm{i})
$$

No segundo nível do modelo são especificados os componentes utilizados na estimação do logaritmo do risco $\theta_{\mathrm{i}}$ :

$$
\log \left(\theta_{i}\right)=\beta_{0}+\beta_{p} X_{i p}+u_{i}+v_{i},
$$

onde $\beta_{0}$ é um parâmetro, $\beta_{\mathrm{p}}=\left(\beta_{1}, \beta_{2}, \ldots, \beta_{\mathrm{k}}\right)$ é um vetor de parâmetros que, quando colocados na função exponencial representam os efeitos de cada uma das $\mathrm{k}$ covariáveis no $\log \left(\theta_{\mathrm{i}}\right)$ - no caso de ser um modelo com inclusão de covariáveis, $X_{\mathrm{ip}}$ é um vetor de covariáveis e $u_{\mathrm{i}}$ e $v_{\mathrm{i}}$ são vetores de efeitos aleatórios. $\mathrm{O}$ componente $u_{i}$ representa um efeito aleatório espacial não estruturado, que pode ser visto como decorrente de particularidades de cada área, ou seja, efeitos de pequena escala que não ultrapassam as fronteiras das áreas. A cada um dos componentes $u_{i}$ será atribuída independentemente uma distribuição $a$ priori normal com média zero e parâmetro de precisão $\tau_{u}$, que em geral é desconhecido e denominado hiperparâmetro, já que é um parâmetro de uma distribuição a priori. O componente $v_{i}$ incorpora a estrutura espacial e captura a influência das áreas vizinhas referentes aos efeitos de larga escala, por meio de uma distribuição a priori espacialmente estruturada mediante a definição de uma matriz de vizinhança entre as áreas. No presente estudo, foi utilizada uma matriz de vizinhança binária, na qual cada elemento assume valor 1 quando as áreas são vizinhas e valor 0 , em caso contrário. Ao componente $v_{i}$, atribui-se uma distribuição a priori denominada CAR (Condicional Autorregressiva) normal. Sendo a matriz de vizinhança do tipo binária, o efeito espacial médio da i-ésima área $v_{i}$ é dado pela média aritmética dos efeitos das áreas vizinhas, e a variância é inversamente proporcional à quantidade de regiões vizinhas. Assim, quanto maior o número de vizinhos, maior é a precisão da estimativa de $v_{i}$. Além disso, a especificação completa da distribuição CAR normal depende de um único parâmetro $\tau_{\mathrm{v}}$ (o inverso da variância de $v_{i}$ ), ao qual também será atribuída uma distribuição a priori gama, analogamente à distribuição $a$ priori para $v_{i}$.

O modelo é construído de modo a relacionar os componentes com o logaritmo de $\theta_{\mathrm{i}}$ e não diretamente com o risco $\theta_{\mathrm{i}}$, usando as inúmeras vantagens que a transformação logarítmica pode proporcionar, como evitar que o modelo estime taxas negativas, dado que a função logaritmo sempre retorna números positivos e, além isso, a interpretação das exponenciais dos parâmetros como riscos relativos em casos nos quais o modelo incorpora covariáveis. Depois de obtidas as estimativas de $\log \left(\theta_{\mathrm{i}}\right)$, aplica-se a função exponencial para a obtenção das estimativas do risco $\theta_{\mathrm{i}}$.

No terceiro nível hierárquico do modelo estão as distribuições a priori para cada um dos parâmetros do modelo com $\beta_{0}, \beta_{\mathrm{p}}, u_{i}$ e $v_{i}$. Em geral, ao parâmetro $\beta_{0}$ se atribui uma distribuição a priori Uniforme $(-\infty ;+\infty)$, e a cada um dos elementos do vetor $\beta p$ se atribui independentemente uma distribuição normal com média igual a zero e parâmetro de dispersão $\tau_{\mathrm{p}}$ o maior possível, de modo que essas distribuições a priori sejam pouco informativas.

A modelagem Totalmente Bayesiana considera que, por pressupor que os hiperparâmetros são quantidades aleatórias, distribuições hiperprioris devem ser especificadas aos mesmos. Ao 
estabelecer uma distribuição a priori para cada hiperparâmetro, melhores estimativas são produzidas para cada área, mais próximas do verdadeiro valor. As estimativas para o método Totalmente Bayesiano para a mortalidade infantil foram obtidas por Kato 16 e Kato et al. 17 pela utilização do programa WinBugs (gratuito e disponível para download em: http://www.mrc-bsu. cam.ac.uk/bugs). Para obtenção das estimativas, foram feitas 150 mil simulações, descartando as 5 mil primeiras iterações (burn-in) e com espaçamento de 50 entre os pontos amostrados (thin), a fim de retirar possíveis autocorrelações na simulação.

No programa computacional TerraView 3.2.0, foram calculadas as estimativas BEG e BEL para cada município, usando como base o número de óbitos esperado para cada município e se valendo das taxas padronizadas e da população do município. A matriz de vizinhança construída e utilizada nas análises foi a do critério de contiguidade, ou seja, usa o valor 1 (um) para os municípios vizinhos e o valor 0 (zero) quando os municípios não têm fronteira adjacente. O programa também foi utilizado para construção de mapas temáticos para a visualização das TMI no Estado do Rio Grande do Sul (os maiores coeficientes com tonalidades mais escuras e os menores com tonalidades mais claras). Foram construídos quatro mapas: um mapa para as taxas brutas, outro para as estimativas BEG, um terceiro para as estimativas BEL e o quarto para as estimativas TB.

Foram calculadas as medidas de tendência central (média e mediana) e de dispersão (amplitude, desvio-padrão) para cada estimador (Taxa Bruta, BEL, BEG e TB), considerando os 496 municípios analisados. Para efetuar as comparações entre as abordagens, também foram gerados gráficos para visualizar o comportamento sequencial das estimativas geradas, tendo como referência para todos os gráficos a ordem crescente das taxas brutas dos municípios. Por fim, as estimativas calculadas foram mapeadas para melhor visualização do padrão de mortalidade infantil no estado, identificando regiões de proteção (baixas taxas) e de risco (altas taxas), mediante a ordenação das CRS.

\section{Resultados}

O número de nascidos vivos por município no Rio Grande do Sul, no período analisado, variou entre 35 e 79.672; o número de óbitos em menores de um ano variou entre 0 e 1072. A TMI geral foi de 15,63 óbitos para cada mil nascidos vivos no período acumulado de 2001 a 2004.
O índice de autocorrelação espacial de Moran foi igual a $0,897(p<0,01)$. Dessa forma, devemos considerar que existe uma correlação espacial significativa nos dados.

Na Tabela 1, estão as estatísticas descritivas para cada método de estimação das TMI no Rio Grande do Sul.

Pelos resultados da Tabela 1, podemos verificar que todos os métodos possuem médias similares, porém podemos observar a alta variabilidade da taxa bruta, com um desvio-padrão de 9,52 óbitos por mil nascidos vivos, valor esse muito superior aos dos demais métodos. As estimativas geradas para a taxa de mortalidade infantil pelo método BEL (variação de 4,09 a 46,67) apresentaram a maior variação em torno da média dentre os métodos de suavização utilizados.

A Figura 1 apresenta os mapas para visualização das TMI para o estado no período analisado, construídos no aplicativo TerraView 3.2.0, um para cada metodologia estudada (Taxa Bruta, BEG, BEL e TB).

Comprova-se por meio da visualização dos mapas na Figura 1, que a Taxa Bruta não é uma boa estratégia para expressar a distribuição espacial da TMI, uma vez que o seu mapa apresenta uma aparência de "colcha de retalhos", não evidenciando de forma clara um padrão espacial para a distribuição das taxas de mortalidade infantil em todo o estado. Já as estimativas obtidas pelos métodos bayesianos mostram mais claramente um padrão regional. Após a suavização, os mapas não mais apresentam municípios com TMI zero, mas sim os municípios incorporam nas suas taxas estimadas o padrão subjacente da região na qual estão inseridos.

A Figura 2 apresenta os gráficos para os valores estimados para as TMI - um gráfico para cada metodologia - que servem para comparar as abordagens estudadas mediante a visualização do comportamento sequencial das estimativas

Tabela 1

Estatísticas descritivas das taxas de mortalidade infantil no Rio Grande do Sul, Brasil, no período acumulado de 2001 a 2004, para os 496 municípios.

\begin{tabular}{lccccc}
\hline Estimativa & Mínimo & Máximo & Mediana & Média & DP \\
\hline Taxa Bruta & 0,00 & 62,50 & 14,86 & 15,63 & 9,52 \\
BEG & 10,10 & 28,16 & 15,44 & 15,70 & 2,17 \\
BEL & 4,09 & 46,67 & 15,11 & 15,71 & 4,49 \\
TB * & 9,96 & 29,89 & 15,96 & 16,16 & 2,98
\end{tabular}

BEG: Bayesiano Empírico Global; BEL: Bayesiano Empírico Local; DP: desvio-padrão; TB: Totalmente Bayesiano.

* Obtida de Kato 16 e Kato et al. 17 
Figura 1

Mapas das taxas de mortalidade infantil (TMI) estimadas pelos métodos Taxa Bruta (1a), Bayesiano Empírico Global (1b), Bayesiano Empírico Local (1c) e Totalmente Bayesiano (1d).

1a) Taxa Bruta

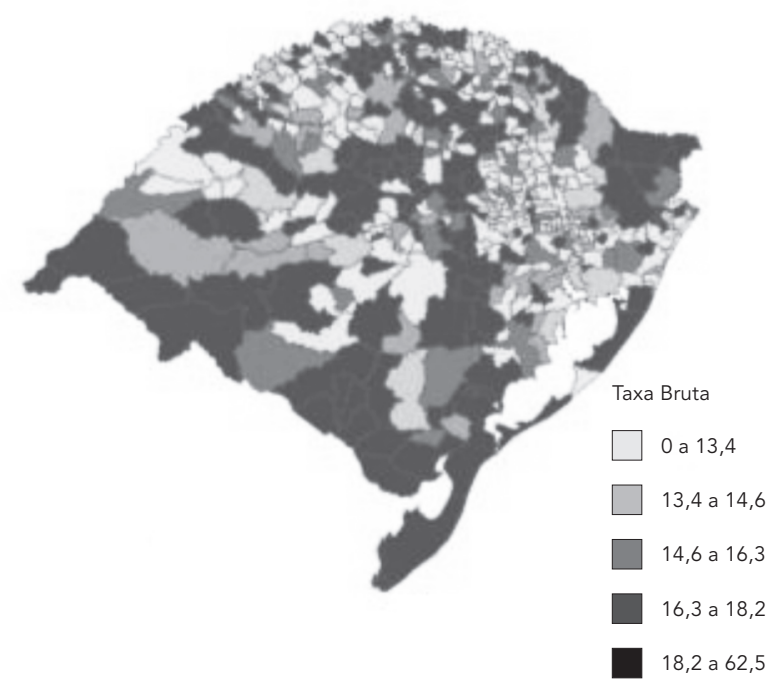

1b) Bayesiano Empírico Global

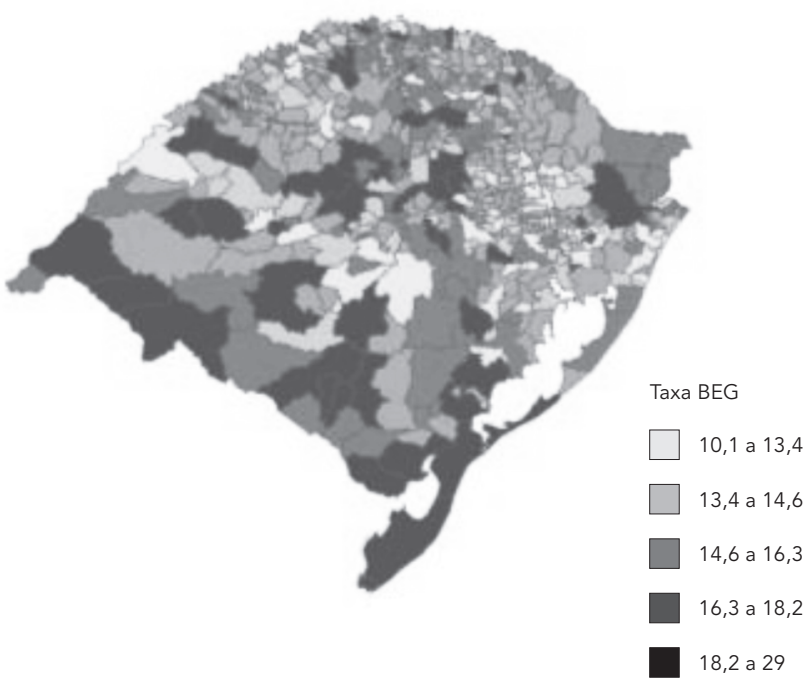

1c) Bayesiano Empírico Local

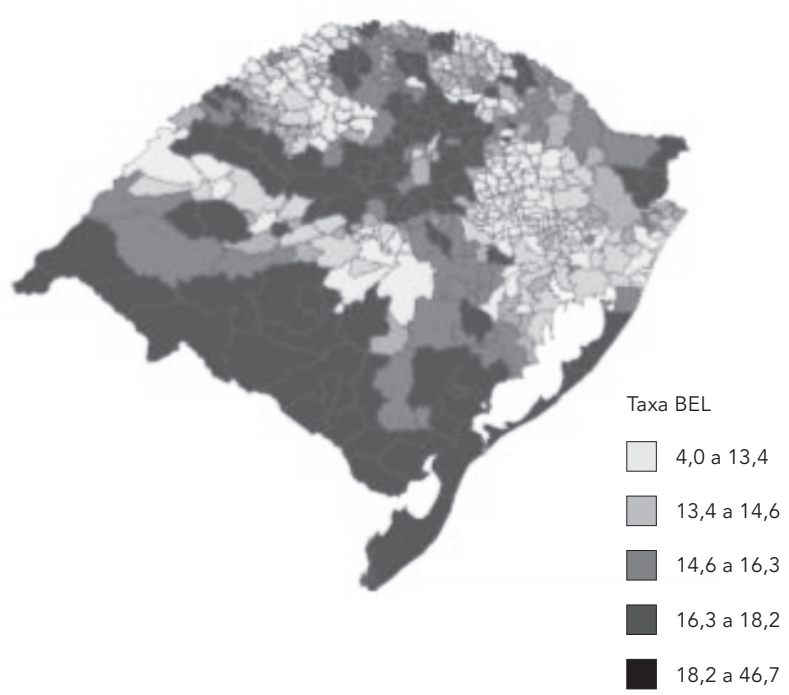

1d) Totalmente Bayesiano

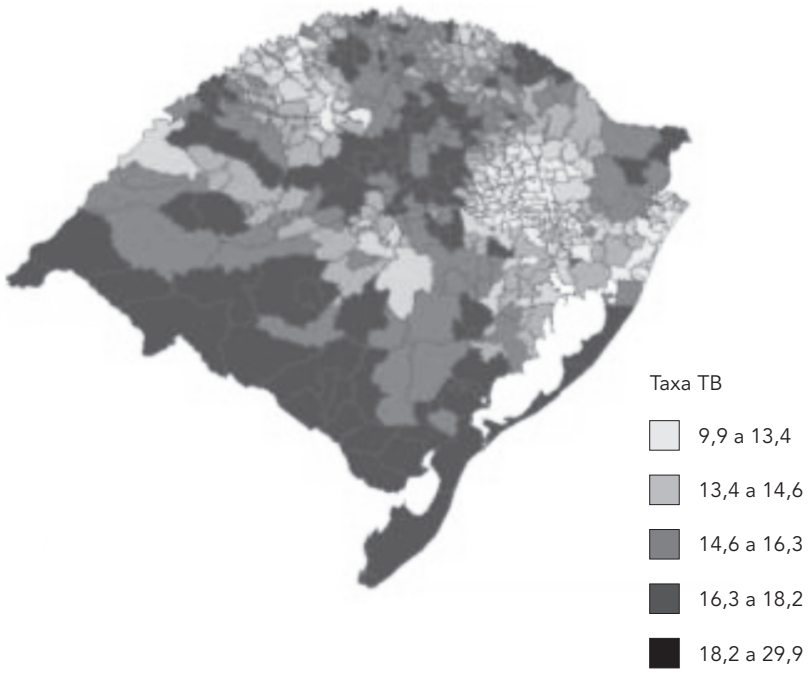

o $\mathfrak{n}^{N}$

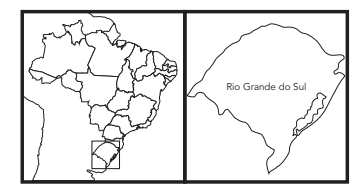

$\stackrel{0}{1} \quad 2 \quad 3$ 
Gráficos de sequência para as estimativas geradas pelos métodos Taxa Bruta (2a), Bayesiano Empírico Global (2b), Bayesiano Empírico Local (2c) e Totalmente Bayesiano (2d).

1a) Taxa Bruta

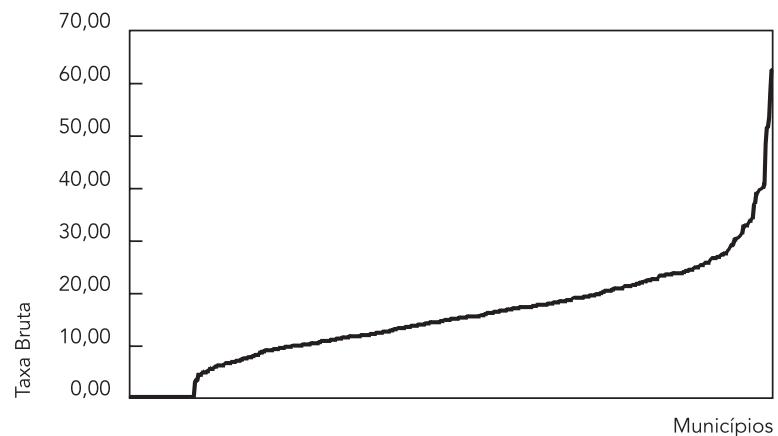

1c) Bayesiano Empírico Local

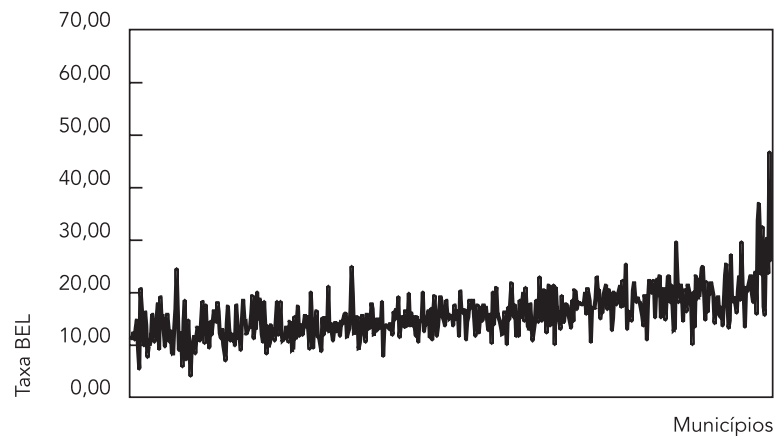

1b) Bayesiano Empírico Global

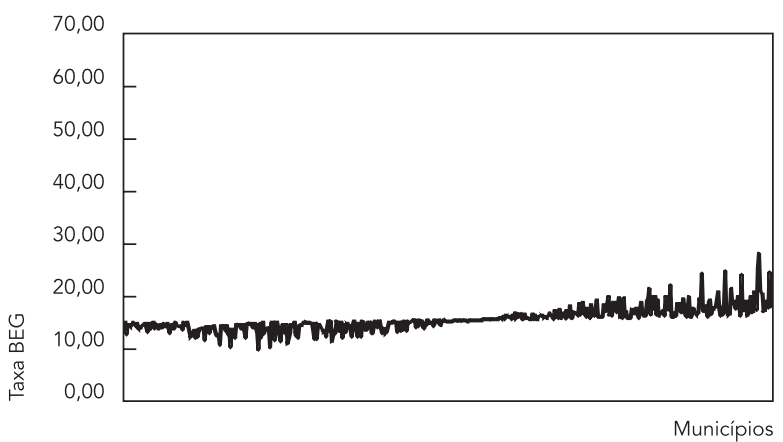

1d) Totalmente Bayesiano

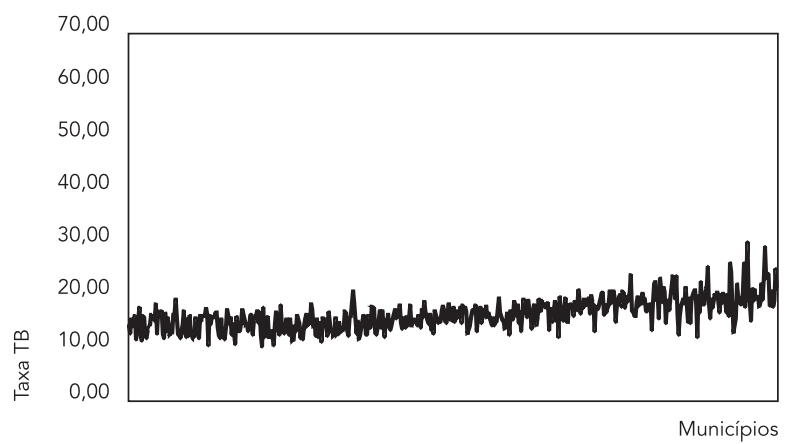

obtidas. Os valores foram organizados segundo a ordem crescente dos valores das Taxas Brutas dos municípios.

Pela análise das Figuras 2c e Figura 2d, pode-se perceber que as abordagens BEL e BEG fornecem estimativas similares aos resultados gerados via estimação TB. Verifica-se um "nó" no gráfico das estimativas BEG, que se explica porque essa metodologia de estimação utiliza a taxa média geral (global) como referência, e a variabilidade das estimativas BEL é maior, isto é, ainda permanecem valores bastante altos em alguns municípios.

Conforme os resultados da Figura 2, podemos concluir que os resultados obtidos pelos métodos BEG e TB são os mais similares, e na Tabela 2 apresenta-se a distribuição das CRS do Rio Grande do Sul pela análise dos quartis das TMI médias por CRS estimadas pelos métodos BEG e
TB. O último quartil corresponde aos valores de TMI mais altos.

Pela Tabela 2, pode-se verificar que o ordenamento das CRS em relação à distribuição das TMI por quartis é praticamente o mesmo, quer utilizando o método BEG quanto utilizando o método TB. Há apenas uma alteração de ordem entre duas CRS nos quartis que mostram as melhores regiões em relação à TMI e, do ponto de vista de gerência e alocação de recursos, os resultados seriam os mesmos, pois as regiões menos favorecidas, isto é, municípios com mais elevadas TMI, têm a mesma classificação.

\section{Discussão e conclusões}

Os resultados corroboram a necessidade do uso de métodos de suavização para melhor repre- 
Distribuição das Coordenadorias Regionais de Saúde (CRS) do Rio Grande do Sul, Brasil. Análise pelos quartis * das taxas de mortalidade infantil (TMI) estimadas pelos métodos Bayesiano Empírico Global (BEG) e Totalmente Bayesiano (TB).

\begin{tabular}{|c|c|c|}
\hline Quartil & BEG & TB \\
\hline 4으 & 3ạ CRS, 7ạ CRS, 9ạ CRS, 10ạ CRS e 15ạ CRS & 3ạ CRS, 7ạ CRS, 9ạ CRS, 10ạ CRS e 15ạ CRS \\
\hline 3으 & 6a $\mathrm{CRS}$, 8a CRS, 12a $\mathrm{CRS}$, 13a CRS e 19a CRS & 6a $\mathrm{CRS}$, 8a CRS, 12a $\mathrm{CRS}$, 13a $\mathrm{CRS}$ e 19a CRS \\
\hline 20 & 2ạ CRS, 4ạ CRS, 11a CRS e 17ạ CRS & 2ạ CRS, 4a $\mathrm{CRS}$, 11a $\mathrm{CRS}$ e 14a $\mathrm{CRS}$ \\
\hline $1 \underline{\text { 으 }}$ & 1ạ CRS, 5ạ CRS, 16ạ CRS, 14ạ CRS e 18ạ CRS & 1a $\mathrm{CRS}, 5 \underline{\text { a }} \mathrm{CRS}, 16 \underline{a} \mathrm{CRS}, 17 \underline{a} \mathrm{CRS}$ e 18a $\mathrm{CRS}$ \\
\hline
\end{tabular}

* O 4o quartil refere-se às piores TMI para o período de 2001 a 2004 no Rio Grande do Sul.

sentar o padrão subjacente dos dados em cada município. Essa é a principal justificativa para o uso da metodologia bayesiana de suavização das taxas para reduzir a alta variação que ocorre em razão de flutuações aleatórias do cálculo bruto das taxas. A alta variabilidade nos valores calculados pela taxa bruta está relacionada ao tamanho da população sob risco e às instabilidades presentes na essência do indicador.

A Tabela 2 evidencia as CRS com maiores valores das TMI ao sudoeste e centro-oeste do estado, e ainda, ao nordeste também foram detectadas regiões de elevados valores para o indicador. A 10a CRS estava desprovida de UTI neonatal até abril de 2009. Sendo assim, foram implantados no ano de 2009 dez leitos de UTI neonatal nessa região. Esse investimento foi demandado devido, basicamente, a óbitos por falta de acesso a leito de cuidado intensivo - óbitos neonatais, precoces, vinculados à prematuridade. A região possui 7 dos 35 municípios prioritários para a redução do óbito infantil no estado. Ainda, algumas regiões do estado que apresentam taxas de mortalidade infantil altas correspondem a municípios com áreas indígenas ou de assentamentos onde há grande vulnerabilidade social, com a ocorrência de óbitos no período infantil tardio, vinculados a fatores de risco conhecidos e identificados nesta população.

Existem diversos aplicativos utilizados para a análise estatística de dados espaciais que já possuem implementado o método de estimação Bayesiano Empírico, o qual pode ser empregado mediante interfaces bem mais acessíveis e amigáveis se comparados com os procedimentos computacionais utilizados para o método de estimação Totalmente Bayesiano. No caso particular do programa TerraView, uma desvantagem é que ele não fornece uma medida da precisão das estimativas geradas, o programa não calcula os intervalos de confiança ${ }^{21}$ para os riscos estimados. Já para as taxas Totalmente Bayesianas, estimadas com o auxílio do aplicativo WinBugs, pode-se obter o cálculo de intervalos de credibilidade para poder medir a precisão das estimativas geradas. Tal aplicativo requer um conhecimento mais apurado de sua linguagem, uma vez que a definição das distribuições a priori e outros passos da análise devem ser realizados por meio de linhas de programação, assim como o do aplicativo R-INLA.

No estudo de Ugarte et al. 5, também foi feito um comparativo entre os dois métodos bayesianos e as estimativas obtidas foram muito similares. Os autores apontam que em casos nos quais se desejam identificar regiões de elevado risco, o excesso de suavização produzida pelo método Totalmente Bayesiano pode ser um problema no que tange a não detectar regiões de potencial risco para o evento analisado.

Neste trabalho, assim como no trabalho de Bernadinelli \& Montonolli 11, os métodos Bayesianos Empíricos apresentaram resultados similares aos apresentados pela abordagem Totalmente Bayesiana. Assim, na falta de recursos computacionais mais sofisticados, e também pela dificuldade de implementação e tempo de computação para geração de estimativas Totalmente Bayesianas, as estimativas Bayesianas Empíricas calculadas pelo uso do programa TerraView são resultados que devem ser considerados úteis para os profissionais da área da saúde - em especial da epidemiologia - para obtenção do padrão subjacente dos eventos estudados, salientando regiões com baixas ou altas taxas do evento de interesse.

A identificação das regiões de elevado risco de mortalidade infantil é um passo importante para uma melhor alocação dos recursos financeiros e humanos necessários à redução de tal indicador pelos gestores em saúde. 


\section{Resumo}

A mortalidade infantil é um sensivel indicador de saúde. Conhecer o seu perfil geográfico auxilia na formulação de estratégias de saúde pública. O mapeamento de doenças tem por objetivo descrever a distribuição geográfica das taxas de mortalidade ou incidência de doenças por intermédio de mapas. Em razão da alta instabilidade das taxas brutas quando há pequenas áreas, utilizam-se os métodos de suavização bayesiana, que se valem de informações de toda a região ou da vizinhança para estimar as taxas. $O$ artigo faz a comparação entre os métodos Bayesiano Empírico e Totalmente Bayesiano para as taxas de mortalidade infantil (dados acumulados de 2001 a 2004) no Rio Grande do Sul, Brasil. O trabalho aponta as vantagens do uso dos estimadores bayesianos na visualização espacial dos mapas. Os métodos Bayesianos Empíricos apresentaram resultados muito semelhantes aos dos métodos Totalmente Bayesianos e possuem a grande vantagem de ser de fácil utilização por profissionais da área de saúde, destacando igualmente os principais padrões espaciais da taxa de mortalidade no Rio Grande do Sul no período estudado.

Mortalidade Infantil; Coeficiente de Mortalidade; Mapa de Risco

\section{Colaboradores}

S. L. C. Silva realizou a revisão da literatura sobre modelagem estatística espacial, discutiu sobre a concepção do artigo e redação do texto final, realizou a análise estatística dos dados e elaborou a escrita inicial do artigo. J. M. G. Fachel orientou o trabalho, participou da concepção do artigo, da revisão e correção do texto e discussão sobre a redação do texto. S. K. Kato forneceu os dados gerados via simulação em seu trabalho anterior, para que a comparação entre as metodologias pudesse ser feita, participou da concepção do artigo e da redação do texto final. S. L. Bassanesi participou da revisão da literatura sobre modelagem estatística espacial, discussão sobre a concepção do artigo e redação do texto.

\section{Agradecimentos}

Aos colegas do Centro Estadual de Vigilância em Saúde (CEVS) do Estado do Rio Grande do Sul, Luciana Sehn, Carmen e Rodrigo pela atenção e fornecimento de informações relevantes para o projeto.

\section{Referências}

1. Monteiro CA, Nazário CL. Evolução da agenda tradicional da saúde pública: mortalidade infantil e materna. Declínio da mortalidade infantil e equidade social: o caso da cidade de São Paulo entre 1973 e 1993. In: Monteiro CA, organizador. Velhos e novos males da saúde no Brasil: a evolução do país e suas doenças. 2a Ed. São Paulo: Editora Hucitec; 2000. p. 173-85.

2. Laurenti R, Jorge MHP, Lebrão ML, Gotlieb SLD. Estatísticas de saúde. São Paulo: EPU/Edusp; 1985.

3. Maia SF, Sousa TRV. Uma investigação dos determinantes da redução da taxa de mortalidade infantil nos estados da Região Nordeste do Brasil. In: I Congresso da Associação Latino-Americana de População. Caxambu: Associação Latino-Americana de População; 2004. p. 1-17.
4. Oliveira LAP, Mendes MMS. Mortalidade infantil no Brasil: uma avaliação de tendências recentes. In: Minayo MCS, organizador. Os muitos Brasis: saúde e população na década de 80 . São Paulo: Editora Hucitec; 1995. p. 291-303.

5. Ugarte MD, Goicoa T, Militino AF. Empirical Bayes and Fully Bayes procedures to detect high-risk areas in disease mapping. Comput Stat Data Anal 2009; 53:2938-49.

6. Assunção RM. Estatística espacial com aplicações em epidemiologia, economia e sociologia. http:// www.est.ufmg.br/leste/publicacoes.htm (acessado em 28/Jan/2008).

7. Gelman A, Carlin JB, Stern HS, Rubin DB. Bayesian data analysis. London: Chapman and Hall; 1997. 
8. Rue H, Martino S. Approximate Bayesian inference for latent Gaussian models by using integrated nested Laplace approximations. J R Stat Soc Series B Stat Methodol 2009; 71:319-92.

9. Martins TG. Aproximações determinísticas para distribuições a posteriori marginais [Dissertação de Mestrado]. Rio de Janeiro: Instituto de Matemática, Universidade Federal do Rio de Janeiro; 2010.

10. Ayçaquer LC, Rodríguez AB, Rodeiro CLV. Análisis espacial de la mortalidad en áreas geográficas pequeñas. El enfoque bayesiano. Rev Cuba Salud Pública 2003; 29:314-22.

11. Bernadinelli L, Montonolli C. Empirical Bayes versus fully Bayesian analysis of geographical variation in disease risk. Stat Med 1992; 11:983-1007.

12. Breslow NE, Clayton DG. Approximate inference in generalized linear mixed models. J Am Stat Assoc 1993; 88:9-25.

13. Richardson S. Spatial models in epidemiological applications. In: Green P, Hjort NL, Richardson S, editors. Highly structured stochastic systems. Oxford: Oxford University Press; 2003. p. 237-59.

14. Aimsworth LM, Dean CB. Approximate inference for disease mapping. Comput Stat Data Anal 2006; 50:2552-70.

15. Vieira DM. Análise espaço-temporal na estimação de taxas de incidência/mortalidade [Dissertação de Mestrado]. Porto Alegre: Faculdade de Medicina, Universidade Federal do Rio Grande do Sul; 2006.
16. Kato SK. Análise de correlação ecológica: uma abordagem inteiramente Bayesiana para a mortalidade infantil no Rio Grande do Sul [Dissertação de Mestrado]. Porto Alegre: Faculdade de Medicina, Universidade Federal do Rio Grande do Sul; 2007.

17. Kato SK, Vieira DM, Fachel JMG. Utilização da modelagem inteiramente bayesiana na detecção de padrões de variação de risco relativo de mortalidade infantil no Rio Grande do Sul, Brasil. Cad Saúde Pública 2009; 25:1501-10.

18. Marshall RJ. Mapping disease and mortality rates using empirical Bayes estimators'. Appl Stat 1991; 40:28-294.

19. Santos AE, Rodrigues AL, Lopes DL. Aplicações de estimadores Bayesianos empíricos para análise espacial de taxas de mortalidade. In: Anais do VII Simpósio Brasileiro de Geoinformática. Campos do Jordão: Instituto Nacional de Pesquisas Espaciais; 2005. p. 300-9.

20. Richardson S, Thomson A, Best N, Elliot P. Interpreting posterior relative risk estimates in diseasemapping studies. Environ Health Perspect 2004; 112:1016-25.

21. Ugarte MD, Goicoa T, Militino AF. Prediction error estimators in empirical Bayes disease mapping. Environmetrics 2008; 19:287-300.

Recebido em 31/Mai/2010

Versão final reapresentada em 30/Dez/2010 Aprovado em 19/Mai/2011 